\title{
Correction to: EBV-miR-BART8-3p induces epithelial-mesenchymal transition and promotes metastasis of nasopharyngeal carcinoma cells through activating NF-KB and Erk1/2 pathways
}

Cheng Lin 1,2, Jingfeng Zong², Wansong Lin³ ${ }^{3}$ Minghui Wang ${ }^{4}$, Yuanji Xu², Rui Zhou', Shaojun Lin², Qiaojuan Guo ${ }^{2}$, Honglin Chen ${ }^{5}$ Yunbin Ye $\mathrm{e}^{3,6}$, Bin Zhang ${ }^{4^{*}}$ and Jianji Pan ${ }^{2^{*}}$

\section{Correction to: Journal of Experimental \& Clinical} Cancer Research (2018) 37:283.

Doi 10.1186/s13046-018-0953-6

Following publication of the original article [1], the authors reported two errors in the article.

- In the caption of Fig. 1c the sentence "The 20 most highly upregulated EBV BART miRNAs identified between NPC specimens and normal nasopharyngeal mucosal specimens" should instead read "The highly upregulated EBV BART miRNAs identified between NPC specimens and normal nasopharyngeal mucosal specimens".

- In Fig. 3a, the first image in the BART8-3p group was inadvertently imported and replaced with the original one in the NC group. A corrected version of Fig. 3 is shown in this Correction.

\footnotetext{
Author details

${ }^{1}$ Fujian Medical University, Fuzhou 350108, Fujian Province, China.

${ }^{2}$ Department of Radiation Oncology, Fujian Cancer Hospital \& Fujian Medical University Cancer Hospital, Fuzhou 350011, Fujian Province, China. 3 Laboratory of Immuno-Oncology, Fujian Cancer Hospital \& Fujian Medical University Cancer Hospital, Fuzhou 350011, Fujian Province, China. ${ }^{4}$ Department of Genetics and Genomic Sciences, Icahn Institute of Genomics and Multiscale Biology, Mount Sinai Center for Transformative Disease Modeling, Icahn School of Medicine at Mount Sinai, 1470 Madison Avenue, New York, NY 10029, USA. ${ }^{5}$ State Key Laboratory for Emerging Infectious Diseases, Department of Microbiology and the Collaborative Innovation

*Correspondence: bin.zhang@mssm.edu; panjianji1956@163.com

${ }^{4}$ Department of Genetics and Genomic Sciences, Icahn Institute of Genomics and Multiscale Biology, Mount Sinai Center for Transformative Disease Modeling, Icahn School of Medicine at Mount Sinai, 1470 Madison Avenue, New York, NY 10029, USA

${ }^{2}$ Department of Radiation Oncology, Fujian Cancer Hospital \& Fujian Medical University Cancer Hospital, Fuzhou 350011, Fujian Province, China Full list of author information is available at the end of the article
}

Center for Diagnosis and Treatment of Infectious Diseases, The University of Hong Kong, Hong Kong, SAR, China. ${ }^{6}$ Fujian Provincial Key Laboratory of Translational Cancer Medicine, Fuzhou 350014, Fujian, China.

Received: 13 December 2018 Accepted: 24 January 2019 Published online: 24 January 2019

\section{Reference}

1. Lin, et al. EBV-miR-BART8-3p induces epithelial-mesenchymal transition and promotes metastasis of nasopharyngeal carcinoma cells through activating NF-kB and Erk1/2 pathways. J Exp Clin Cancer Res. 2018;37(283). https://doi. org/10.1186/s13046-018-0953-6.

(c) The Author(s). 2019 Open Access This article is distributed under the terms of the Creative Commons Attribution 4.0 International License (http://creativecommons.org/licenses/by/4.0/), which permits unrestricted use, distribution, and reproduction in any medium, provided you give appropriate credit to the original author(s) and the source, provide a link to the Creative Commons license, and indicate if changes were made. The Creative Commons Public Domain Dedication waiver (http://creativecommons.org/publicdomain/zero/1.0/) applies to the data made available in this article, unless otherwise stated. 


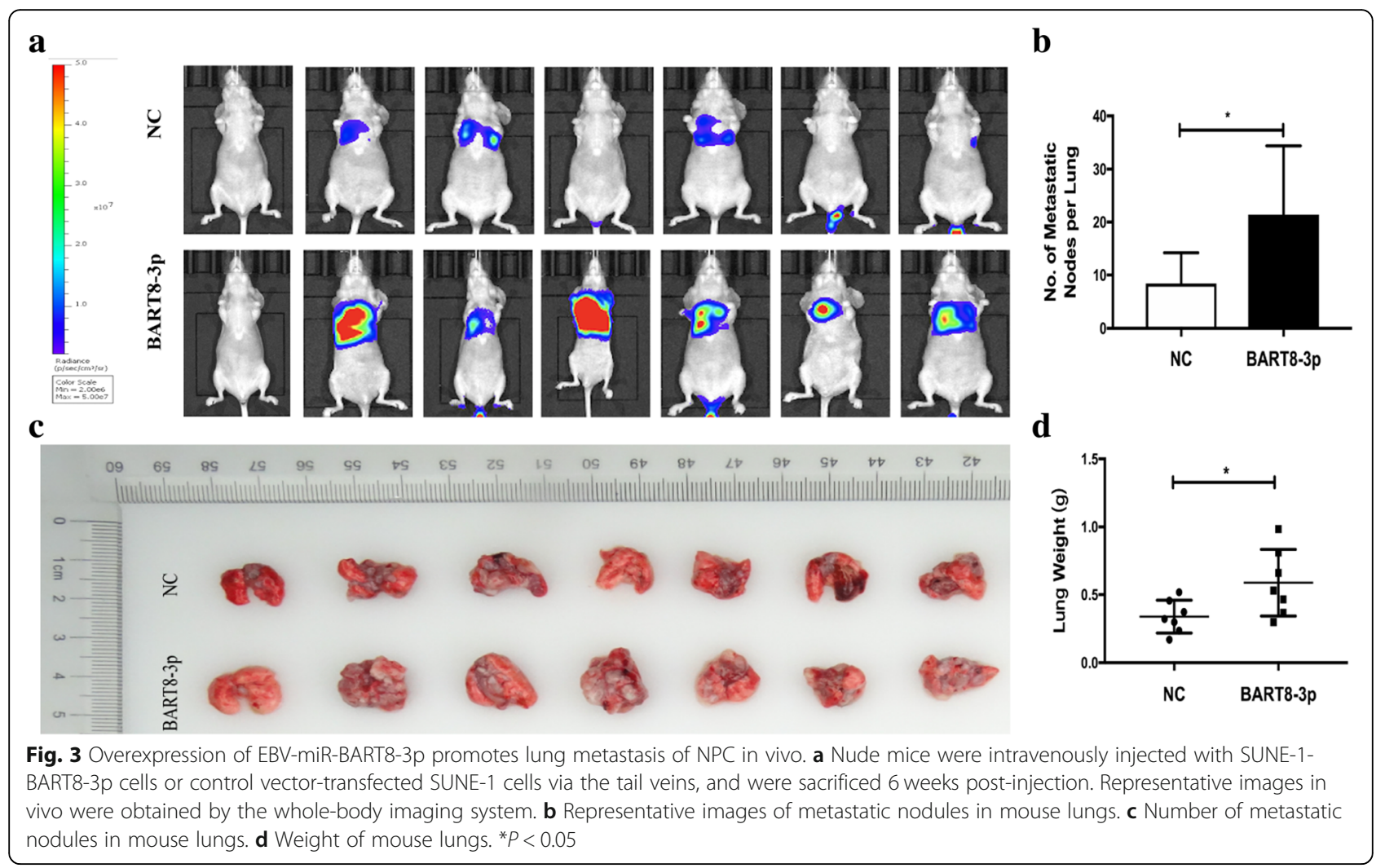

